\title{
The Integration of Emotions in Memories: Cognitive-emotional Distinctiveness and Posttraumatic Stress Disorder
}

\author{
ADRIEL BOALS ${ }^{1 *}$ and DAVID C. RUBIN ${ }^{2,3}$ \\ ${ }^{1}$ Department of Psychology, University of North Texas, USA \\ ${ }^{2}$ Department of Psychology and Neuroscience, Aarhus University, Denmark \\ ${ }^{3}$ Center on Autobiographical Memory Research, Aarhus University, Denmark
}

\begin{abstract}
Summary: The current study examined cognitive-emotional distinctiveness (CED), the extent to which emotions are linked with event information, in memories associated with PTSD. Participants either with PTSD $(n=68)$ or without PTSD $(n=40)$ completed a modified multidimensional scaling technique to measure CED for their most negative and most positive events. The results revealed that participants in the PTSD group evidenced significantly lower levels of CED. This group difference remained significant when we limited the analysis to traumatic events that led to a PTSD diagnosis $(n=33)$ in comparison to control participants who nominated a traumatic event that did not result in $\operatorname{PTSD}(n=32)$. Replicating previous findings, CED levels were higher in memories of negative events, in comparison to positive events. These results provide empirical evidence that memories associated with PTSD do contain special organizational features with respect to the links between emotions and memory. Implications for understanding and treating PTSD are discussed. Copyright (C) 2010 John Wiley \& Sons, Ltd.
\end{abstract}

Approximately $7.8 \%$ of people who experience a potentially traumatic event develop PTSD (Kessler, Berglund, Demler, Jin, \& Walters, 2005). An argument can be made that if PTSD symptoms persist after the event itself has ended, we can assume that memory, in one form or other, is contributing to the maintenance of PTSD (Rubin, Berntsen, \& Bohni, 2008). In this sense, memories of traumatic experiences help to maintain PTSD symptoms (Brewin, Dalgleish, \& Joseph, 1996; Foa, Molnar, \& Cashman, 1995; Rubin, Feldman, \& Beckham, 2003). Therefore, an examination of memories of potentially traumatizing events has the potential to yield valuable insights into the nature and possible treatment of PTSD.

Some researchers have argued that memories of traumatic events differ from everyday memories in that they have unique structural and organizational features. For instance, some argue that memories associated with PTSD fail to become appropriately integrated with other autobiographical memories (Ehlers \& Clark, 2000), lack coherence (Barclay, 1995), and are fragmented and poorly organized (Foa \& Riggs, 1993; van der Kolk \& Fisler, 1995). However, others have argued that such memories do not differ in kind from non-traumatic memories (Berntsen, Willert, \& Rubin, 2003; Porter \& Birt, 2001; Shobe \& Kihlstrom, 1997). For example, Rubin, Boals, and Berntsen (2008) recently demonstrated that no special mechanisms of traumatic memory are necessary to account for PTSD. Rather, basic mechanisms of emotion and memory can be used to understand PTSD. There is also evidence that the extent to which a trauma event has become integrated into the life story is positively related to PTSD symptoms. Individuals who constructed their trauma event as central to their identity and used their trauma experience to colour how they interpret new experiences reported higher levels of PTSD symptoms and depression (Berntsen \& Rubin, 2006). Although there is great controversy surrounding the exact nature of traumatic memory structure, most researchers would agree that organizational features of traumatic

*Correspondence to: Adriel Boals, Department of Psychology, University of North Texas, Box 311280, Denton, TX 76203, USA. E-mail: adriel@unt.edu memories contribute to the onset and maintenance of PTSD symptoms and serve as a formidable barrier to recovery (see Dalgleish, 2004 for a review).

Most researchers agree that emotion is a central organizational aspect of trauma memories (Brewin \& Holmes, 2003; Stein, Trabasso, \& Albro, 2001). The presence of negative emotions associated with a trauma memory is predictive of future psychopathology and PTSD symptoms (Ehlers, Mayou, \& Bryant, 1998; Talarico \& Rubin, 2003) and may undermine subsequent attempts at therapy (Foa, Riggs, Massie, \& Yarczower, 1995). In the current paper, we posit that a key distinguishing organizational feature of traumatic memories that result in PTSD is the extent to which emotions have been linked with the less emotional aspects of the memory (see Conway \& Pleydell-Pearce, 2000). The idea of examining organizational features of traumatic memories associated with PTSD is not novel. Foa and Kozak (1986) have proposed a fear network model of traumatic memories, which emphasizes the links between representations of the trauma event and affective responses. A strong integration of memory and emotion should lead any explicit or implicit thought about the trauma event, whether voluntary or involuntary, to automatically activate the associated emotions (Boals, Rubin, \& Klein, 2008). If the emotions associated with the memory are of high emotional intensity, the combination of frequent activation and high emotional intensity can give rise to PTSD symptoms. The strong emotional associations with the traumatic memory keep the memory highly available, leading to further rehearsal. The rehearsal in turn maintains the memory and its emotional impact over long periods of time.

One methodology that can be used to empirically explore the degree of integration of emotions in a memory is multidimensional scaling (MDS). MDS is a mathematical tool that can also be used to reveal organizational associations between items related to a particular domain (Schiffman, Reynolds, \& Young, 1981). In MDS, participants make dissimilarity ratings between stimuli related to a particular event or concept. The dissimilarity ratings are then used to 
determine the underlying organization and representation of the concepts within the context of the event. If the stimuli being rated include the event and its associated emotions, we can measure the strength of the associations or links between the event and its associated emotions. This measure is referred to as cognitive-emotional distinctiveness (CED; Boals \& Klein, 2005; Boals et al., 2008).

CED comes from an association network model of memory (see Anderson, 1983; Bower, 1981) and refers to the strength of the link between emotion nodes and event nodes. Emotion nodes are viewed as playing a central role in organizing memory (Bower, 1981). Understanding the connections involving emotion nodes is critical to understanding pathology (Yates \& Nasby, 1993). An event with a high level of CED has a clear separation between the event and emotion nodes (relatively weak links). In this situation, when an individual thinks about or is reminded of the event, there is a relatively low likelihood of an automatic activation of the associated emotions. However, in events with a low level of CED, the event and emotion nodes are not separated, but rather are integrated with each other (relatively strong links). Similar to a spread of activation model, when the event nodes become active, the associated emotions are automatically activated. If the emotion nodes are of high emotional intensity, this automatic activation of the emotion nodes leads to an uncontrollable and emotionally intense distress response. The individual will have difficulty thinking about or being reminded of the event without automatically triggering a simultaneous distress response. This inability to think about the event without being flooded with emotions may hinder subsequent recovery attempts and contribute to the maintenance of PTSD symptoms. Hence, we hypothesize that low CED levels are an important organizational feature of memories associated with PTSD.

Following this logic, it would be advantageous to have relatively weak links between event and emotion nodes (high CED levels) in memories of negative events because this memory feature would protect individuals from the repeated experiencing of unwanted distress reactions. But for positive events, there is no harm in an automatic activation of the associated positive emotions. In support of this argument, Boals et al. (2008) found that memories of negative events, on average, have higher CED levels than memories of positive events. If CED is an adaptive coping mechanism to help individuals not experience repeated distress responses, then we would expect that memories with the triple combination of (1) negative valence, (2) high emotional intensity and (3) low CED levels should be related to a pattern of distress and PTSD symptoms. Because such memories have low CED levels, activation of the event nodes automatically activates the emotion nodes. If the associated emotions are both negative and emotionally intense, they may be 'hot' and automatically trigger an emotionally intense, negative response. In support of this argument, recent studies have revealed that memories that have the aforementioned three features are associated with elevated levels of reliving, visceral emotional responses, PTSD symptoms, and worse overall mental health (Boals et al., 2008).

Although CED appears to be an important organizational feature of stressful memories, CED levels have yet to be examined in persons with PTSD. All previous studies examining CED asked participants to nominate 'a very negative event' from their lives. Although some of the nominated memories would meet the DSM-IV trauma criteria, the majority of the nominated events were stressful, but non-traumatic (e.g. 'romantic breakup') that would fail to produce a formal PSTD diagnosis. The purpose of the current study is to compare CED levels in trauma memories in people with and without PTSD. Our primary hypothesis is that CED levels in memories of traumatic experiences associated with PTSD will have lower levels of CED (stronger links between event and emotion nodes) than memories of traumatic events not associated with PTSD. Such a finding would further support the theory that CED levels are a key organizational memory feature associated with PTSD. In addition, we will attempt to replicate the findings of lower levels of CED in positive events, in comparison to negative events, using a PTSD sample.

\section{METHODS}

\section{Participants}

Participants were recruited via flyers posted in a Veterans Affairs Medical Center in the southeastern United States, as well as through invitations to participants in other studies examining trauma and PTSD. Participants were screened for enrolment in a larger study. Any potential participant who met DSM-IV criteria for organic mental disorder, schizophrenia, current manic syndrome, or current alcohol or substance abuse/dependence was excluded. In addition, the data from four participants in the PTSD group and one participant in the control group were lost due to a computer error. A total of 122 participants with current PTSD $(n=80)$ and without PTSD $(n=42)$ were recruited. All participants in the PTSD group had a clinical diagnosis of PTSD using the Clinician Administered PTSD Scale (CAPS), whereas no participants in the control group had a diagnosis, nor current subthreshold PTSD. The CAPS was administered by a Masters level clinical psychologist under the supervision of a licensed clinical psychologist. Common events that resulted in the PTSD diagnosis include rape, physical abuse, childhood sexual abuse, witnessing murder, and severe car accidents. All analyses were conducted on de-identified data from a total of 108 participants with current PTSD $[n=68$ (41 female), average age $=47.1(S D=10.0)$ years, average years of education $=13.4(S D=2.4)]$ and without PTSD $[n=40$ (21 female), average age $=45.5(S D=12.0)$ years, average years of education $=14.6(S D=3.1)]$. There were no differences on these demographic variables (both $t$ 's > .10).

\section{Materials}

\section{$C E D$}

CED is measured using a modified multidimensional scaling (MDS) technique (Boals \& Klein, 2005; Boals et al., 2008). A single set of stimuli is chosen to represent a concept and participants make dissimilarity ratings between all possible pairs of these stimuli. The result is a model that depicts the 
organizational structure of the stimuli, including which stimuli cluster together.

The modified technique involves participants nominating an event (e.g. 'my day at the beach'), then nominating the stimuli that comprise that event (e.g. 'swimming in the water' and 'building a sandcastle'), including associated emotions (e.g. 'happy'). CED is measured by calculating the mean rating of the event-emotion pairs (e.g. 'swimming in the water' - 'happy'). Higher dissimilarity ratings of eventemotion pairs reflect weaker links between event nodes and associated emotion modes, hence higher CED levels; lower dissimilarity scores reflect greater integration between the event and the associated emotions, hence lower CED levels. Mean dissimilarity ratings of event-event (e.g. 'swimming in the ocean'-'building a sandcastle') pairs are calculated for comparison purposes. Comparisons of event-event pair ratings are important to demonstrate that any group differences are due to links between event and emotion nodes, and not due to respondent rating tendencies. The modified MDS procedure results in 28 event-event pairs, 32 event-emotion pairs and six emotion-emotion pairs to be rated for each memory. This measurement technique produces similar individual differences measures produced by traditional MDS. More specifically, the mean ratings for event-emotion pairs are highly correlated with normalized dimension weights produced by INDSCAL, but unlike traditional MDS, can be applied to idiosyncratic events (see Boals et al., 2008). This latter feature is critical if one wants to apply MDS to individual traumatic memories.

In reference to a selected memory, participants were instructed to '...think about items (such as persons, places, thoughts and events) that you believe are central to your memory for this event. Then, in the spaces provided below, please list eight of these items and four emotions that you believe are most central to your memory for this event'. All possible non-repeating pairs of the 12 stimuli $(n=66)$ were presented on a computer screen. Participants rated the pairs on a scale from 0 (exactly same) to 50 (completely different).

\section{Procedure}

As part of a larger study, participants nominated their trauma event, their next two most negative events from their lives, and their three most positive events from their lives. Hence participants nominated their three most negative and three most positive events. Of these six nominated events, participants were then asked to select 'their most negative event' and 'their most positive event'. They then completed the CED measurement for these latter two events.

\section{RESULTS}

The age of the participants was entered as a covariate and did not result in any significant effects; hence this variable was excluded from all analyses. The average time since trauma in the PTSD group was $m=15$ years, 8 months ( $S D=14$ years) and in the control group was 15 years, 9 months ( $S D=14$ years).

To test our two hypotheses, a 2 (PTSD versus non-PTSD group) $\times 2$ (valence) ANOVA with a repeated measures factor on the valence variable was conducted on the CED scores. Consistent with our hypothesis, there was a main effect of valence, $F(1,106)=6.71, p<.05, \eta^{2}=.059$. CED scores for negative events $(m=20.4, S D=11.2)$ were significantly higher than for positive events $(m=17.6$, $S D=11.8)$. Consistent with our other hypothesis, there was a main effect of group on CED scores, $F(1,106)=3.99$, $p<.05, \eta^{2}=.036$. CED scores for the PTSD groups were significantly lower $(m=17.6, S D=9.8)$ than CED scores for the control group $(m=21.6, S D=10.0)$. The interaction term was non-significant $F<1$, indicating that the PTSD group evidenced equivalently lower CED scores for their negative and positive event.

For comparison purposes, a similar 2 (group) $\times 2$ (valence) ANOVA was conducted on mean scores for event-event pairs. There were no main effects of group or valence, or any significant interaction, all $p s>.15$. An alternative explanation of the main effect of valence is that there is a greater range of negative than positive emotions, so greater dissimilarity for negative events may just mean that different moments of the negative events triggered different negative emotions, leading to greater dissimilarity ratings. Thus we examined participants' ratings of emotion-emotion pairs for positive and negative events. A paired $t$-test analysis revealed no significant differences of participants' mean dissimilarity ratings of emotion-emotion pairs between positive events $(m=15.0, S D=14.4)$ and negative events $(m=17.1, S D=14.6), t(107)=1.60, p>.10$.

Although the previous set of results is consistent with the hypotheses, the primary hypothesis is specifically concerned with traumatic memories associated with PTSD. Participants were asked to complete the CED measure for 'their most negative event'. Some of the participants in the PTSD group selected the event that resulted in their PTSD diagnosis $(n=33)$; others selected one of their other two nominated negative events $(n=35)$. In addition, some of participants in the control nominated a negative event that meets the DSMIV-R A criterion for a trauma $(n=32)$, whereas others nominated a negative event that fails to meet the A criterion $(n=8)$. We believe that the fairest test of our hypothesis would be to compare only events in the PTSD group that we confirmed were the events that resulted in their PTSD diagnosis (trauma events associated with PTSD) to only events in the control group that meet the A criterion for a trauma event (trauma events NOT associated with PTSD). These two groups did not significantly differ on the variables of sex, age, years of education or time since event. The results confirmed our prediction. Trauma events associated with PTSD evidenced significantly lower levels of CED $(m=16.4, S D=10.5)$ than did trauma events from the control group $(m=22.4, S D=10.5), t(64)=2.30, p<.05$, Cohen's $d=.57$. For comparison purposes, similar analyses revealed no significant differences between these two groups on mean ratings of event-event pairs for the trauma event, CED scores for their positive events, and event-event pairs for their positive event, all $p$ 's $>.10$.

\section{DISCUSSION}

The results from the current study supported our primary hypothesis that CED levels in memories of traumatic 
experiences associated with PTSD will have lower levels of CED (stronger links between event and emotion nodes) than memories of traumatic events not associated with PTSD. Participants with PTSD had lower CED scores than did participants without PTSD. As a more direct test of our primary hypothesis, we conducted an additional analysis in which we limited our sample to just the traumas that led to the PTSD diagnosis in the PTSD group and to traumas that could have led to a diagnosis in the group without PTSD. Trauma memories in the group with PTSD exhibited lower levels of CED in comparison to trauma memories in the group without PTSD. These results suggest that CED levels are one way in which people with PTSD differ from those without. The particularly strong links between event and emotion nodes in the PTSD group likely contributes to the intense and uncontrollable affective responses commonly associated with PTSD. Activation of negative events that have relatively high levels of CED in the control group is less likely to automatically trigger emotional responses. However, we hypothesized that this result would be obtained for negative events, but not for positive events. In the current study, participants with PTSD evidenced lower CED scores for their nominated memories, regardless of the valence of the memory. This finding suggests that individuals with PTSD may integrate emotions and details of events more so than individuals without PTSD, regardless of valence of the event. The lack of a group by valence interaction suggests that this difference in the groups may extend to positive memories as well, but here there should be no negative effects of clinical significance.

The results from the current study also replicated previous findings that CED levels tend to be higher in negative events, in comparison to positive events. We argue that elevated CED levels for negative events is an adaptive coping mechanism that keeps the individual from continually re-experiencing negative emotions. If the links between the event and the emotion nodes are relatively weak, then activation of the event is less likely to result in the automatic activation of the associated emotions, perhaps allowing the individual regulation over their emotional reactions. The current study was the first to test for this valence difference using a PTSD sample. There was no significant interaction between the valence differences and PTSD status. Although the PTSD group had lower CED levels, traumatic memories associated with PTSD have higher CED levels than positive events.

The concept of CED is consistent with associative network models of PTSD. For example, in Foa and Kozak's (1986) fear network model of PTSD, memories and reminders of the trauma become integrated into a fear network. Activation of any stimulus or element of the network automatically triggers a fear response. In essence, the model proposes that strong links between stimuli related to the traumatic experience and fear is central to PTSD. Using a modified MDS technique, we were able to empirically measure the strength of the links between the trauma stimuli and the associated emotions. Consistent with predictions that would be made from a fear network model of PTSD, these links were significantly stronger in memories associated with PTSD.

High CED scores result in less re-experiencing of traumarelated emotions, which is similar to the concept of dissociation. Although similar on the surface, high CED levels and dissociation differ in important ways. First, dissociation is a complex concept with a long theoretical history (Bryant, 2007). In contrast, CED is a more narrowly defined specific measure of links between nodes in a memory network. In dissociation, all aspects of the trauma memory are susceptible to distortion, including perceptual alterations, memory impairment, time distortions, hallucinations and emotional numbing (Cardena \& Spiegel, 1993). Thus we would expect higher ratings of dissimilarity for not only event-emotion pairs but also event-event pairs. In the current study, we found no differences in ratings of event-event pairs as a function of PTSD. To the extent that event-event pair ratings measure dissociation, the current results adds to a growing body of literature that dissociation is not a prominent aspect of PTSD (Bryant, 2007; Wittmann, Moergeli, \& Schnyder, 2006; Zoellner, Jaycox, Watlington, $\&$ Foa, 2003). Second, dissociation is related to hyperarousal and extreme fear and anxiety (Bryant, 2007). The emotional numbing component of dissociation presumes that emotions are being activated, but the intensity and experiencing of these emotions is distorted and dulled. Indeed, in dissociative PTSD, there is greater activation of prefrontal activity to conscious fear and enhanced activity in limbic networks to non-conscious fear (Felmingham et al., 2008). In memories with high CED, the emotions are rarely activated to begin with, so there is no need to dull emotional responses. Third, dissociation is related to greater PTSD symptoms, but high CED is related to lower PTSD symptoms. We believe that individuals who exhibit high CED levels experience fewer PTSD symptoms because the links between the event and emotions in the stressful memory are weak.

The current findings are significant because they provide empirical evidence that memories associated with PTSD do indeed contain specific organizational and structural features with respect to the integration of emotions into the memory, features we have observed in negative memories in general. The differential CED levels do not suggest that PTSD traumatic memories are necessarily 'more fragmented' as has been suggested by some researchers. The CED should not be considered a measure of fragmentation. Rather, the links between event nodes and emotion nodes in such memories are particularly strong. Further, our results do not address whether traumatic memories differ from other types of negative memories. In the current study, we compared traumatic memories associated with PTSD with traumatic memories not associated with PTSD. Past studies that examined CED levels in negative, but non-traumatic memories (e.g. romantic relationship breakups, losing an election) similarly revealed a negative relationship between CED levels and PTSD symptoms (Boals \& Klein, 2005; Boals et al., 2008). Thus our findings do not appear to be confined to just memories of traumatic events. Rubin, Boals, et al., (2008b) claim that people with high PTSD symptoms experience greater affective intensity for all events. Consistent with this finding, in the current study we found that the PTSD group evidenced not only lower CED levels for the trauma event but also their nominated positive event. Thus as negative events become more emotionally intense, lower CED levels have an additive effect, regardless of 
whether the event can be considered as traumatic or not (Boals \& Schuettler, 2009).

There are a few limitations worthy of note. First, an alternative explanation for the results of the current study is that people with PTSD have trouble generating events and emotions and making dissimilarity ratings due to difficulties with concentration, overgeneral memories and avoidance. Although it is important to note that differences between the PTSD and control group were only obtained for the eventemotions pairs, while no group differences were obtained for the event-event pairs. Second, it should be noted that the current results are based on self-reported connections between events and emotions and no physiological measures of emotional reactivity were collected. Lastly, due to the crosssectional nature of the study, we are unable to draw conclusions concerning the causality between CED levels and PTSD.

The combined results from the current study and the studies reported in Boals et al. (2008) suggest that memories associated with PTSD tend to have the triple combination of being negative, emotionally intense and low CED, although other factors may also be involved. In theory, any therapy that can affect any of these three features, which can lead to the highly negative emotions, should be effective treatments for PTSD. One way to view cognitive therapies is that they are effective in part because they can alter the first feature (Yadin \& Foa, 2007). One way to view exposure therapies is that they are effective in part because they can reduce the second feature (Institute of Medicine, 2008). Our data suggest a new possibility - a therapy that can effectively increase CED levels should also be an effective treatment for PTSD. For example, classical conditioning plays a central role in PTSD (Keane, Marshall, \& Taft, 2006). Through habituation, the links between the event and the emotional reaction become weakened, thus increasing CED levels. Note that the weakened links can occur with or without a concomitant reduction in emotional intensity. If either the intensity of the associated emotions or the links between the event and emotion nodes in a traumatic memory can be weakened, then uncontrollable emotional reactions become less likely, thereby reducing the likelihood of PTSD. Hence various types of therapy for PTSD can be effective, as long as the therapy disrupts any aspect of the earlier stated triple combination of negative valence, high emotional intensity and low CED levels.

\section{ACKNOWLEDGEMENTS}

The authors thank National Institute of Mental Health grant number R01 MH066079 and NRSA F32-MH066569 for support. They also thank Gustavo Araujo, Jean C. Beckham and Michelle E. Feldman Dennis for their help.

\section{REFERENCES}

Anderson, J. R. (1983). A spreading activation theory of memory. Journal of Verbal Learning and Verbal Behavior, 22, 261-295.

Barclay, C. R. (1995). Autobiographical remembering: Narrative constraints on objectified selves. In D. C. Rubin (Ed.), Remembering our past:
Studies in autobiographical memories. New York: Cambridge University Press.

Berntsen, D., \& Rubin, D. C. (2006). The centrality of event scale: A measure of integrating a trauma into one's identity and its relation to posttraumatic stress disorder symptoms. Behaviour Research and Therapy, 44, 219-231.

Berntsen, D., Willert, M., \& Rubin, D. C. (2003). Splintered memories or vivid landmarks? Qualities and organization of traumatic memories with and without PTSD. Applied Cognitive Psychology, 17, 675-693.

Boals, A., \& Klein, K. (2005). Cognitive-emotional distinctiveness: Separating emotions from non-emotions in the representation of a stressful memory. Memory, 13, 638-648.

Boals, A., Rubin, D. C., \& Klein, K. (2008). Memory and coping with stress: The relationship between cognitive-emotional distinctiveness, memory valence, and distress. Memory, 16, 637-657.

Boals, A., \& Schuettler, D. (2009). PTSD symptoms in response to traumatic and non-traumatic events: The role of respondent perception and the A2 criterion. Journal of Anxiety Disorders, 23, 458-462.

Bower, G. (1981). Mood and memory. American Psychologist, 36, 129-148.

Brewin, C. R., Dalgleish, T., \& Joseph, S. (1996). A dual representation of posttraumatic stress disorder. Psychological Review, 103, 670-686.

Brewin, C. R., \& Holmes, E. A. (2003). Psychological theories of posttraumatic stress disorder. Clinical Psychology Review, 23, 339-376.

Bryant, R. A. (2007). Does dissociation further our understanding of PTSD? Journal of Anxiety Disorders, 21, 183-191.

Cardena, E., \& Spiegel, D. (1993). Dissociative reactions to the San Francisco Bay Area earthquake of 1989. American Journal of Psychiatry, 150, 474-478.

Conway, M. A., \& Pleydell-Pearce, C. W. (2000). The construction of autobiographical memories in the self-memory system. Psychological Review, 107, 261-288.

Dalgleish, T. (2004). Cognitive approaches to posttraumatic stress disorder: The evolution of multirepresentational theorizing. Psychological Bulletin, 130, 228-260.

Ehlers, A., \& Clark, D. M. (2000). A cognitive model of posttraumatic stress disorder. Behaviour Research and Therapy, 38, 319-345.

Ehlers, A., Mayou, R. A., \& Bryant, B. (1998). Psychological predictors of chronic posttraumatic stress disorder after motor vehicle accidents. Journal of Abnormal Psychology, 107, 508-519.

Felmingham, K., Kemp, A. H., Williams, L., Falconer, E., Olivieri, G., Peduto, A., et al. (2008). Dissociative responses to conscious and nonconscious fear impact underlying brain function in post-traumatic stress disorder. Psychological Medicine, 38, 1771-1780.

Foa, E. B., \& Kozak, M. J. (1986). Emotional processing of fear: Exposure to corrective information. Psychological Bulletin, 99, 20-35.

Foa, E. B., Molnar, C., \& Cashman, L. (1995). Change in rape narratives during exposure to therapy for posttraumatic stress disorder. Journal of Traumatic Stress, 8, 675-690.

Foa, E. B., \& Riggs, D. S. (1993). Posttraumatic stress disorder in rape victims. In J. Oldham, M. B. Riba, \& A. Tasman (Eds.), American psychiatric press review of psychiatry (Vol. 12, pp. 273-303). Washington DC: American Psychiatric Press.

Foa, E. B., Riggs, D. S., Massie, E. D., \& Yarczower, M. (1995). The impact of fear activation and anger on the efficacy of exposure treatment for posttraumatic stress disorder. Behavior Therapy, 26, 487-499.

Institute of Medicine. (2008). Treatment of posttraumatic stress disorder: An assessment of the evidence. Washington, DC: The National Academies Press.

Keane, T. M., Marshall, A. D., \& Taft, C. T. (2006). Posttraumatic stress disorder: Etiology, epidemiology, and treatment outcome. Annual Review of Clinical Psychology, 2, 161-197.

Kessler, R. C., Berglund, P., Demler, O., Jin, R., \& Walters, E. E. (2005). Lifetime prevalence and age-of-onset distributions of DSM-IV disorders in the National Comorbidity Survey Replication. Archives of General Psychiatry, 62, 593-602.

Porter, S., \& Birt, A. R. (2001). Is traumatic memory special? A comparison of traumatic memory characteristics with memory for other emotional life experiences. Applied Cognitive Psychology, 15, 101-117.

Rubin, D. C., Berntsen, D., \& Bohni, M. K. (2008). A memory-based model of posttraumatic disorder: Evaluating basic assumptions underlying the PTSD diagnosis. Psychological Review, 115, 985-1011. 
Rubin, D. C., Boals, A., \& Berntsen, D. (2008). Memory in posttraumatic stress disorder: Properties of voluntary and involuntary, traumatic and non-traumatic autobiographical memories in people with and without PTSD symptoms. Journal of Experimental Psychology: General, 137, 591-614.

Rubin, D. C., Feldman, M. E., \& Beckham, J. C. (2003). Reliving, emotions and fragmentation in the autobiographical memories of veterans diagnosed with PTSD. Applied Cognitive Psychology, 18, 17-35.

Schiffman, S. S., Reynolds, M. L., \& Young, F. W. (1981). Introduction to multidimensional scaling. New York: Academic Press.

Shobe, K. K., \& Kihlstrom, J. F. (1997). Is traumatic memory special? Current Directions in Psychological Science, 6, 70-74.

Stein, N. L., Trabasso, T., \& Albro, E. R. (2001). Understanding and organizing emotional experience: Autobiographical accounts of traumatic events. Empirical Studies of the Arts, 19, 111-130.

Talarico, J. M., \& Rubin, D. C. (2003). Confidence, not consistency, characterizes flashbulb memories. Psychological Science, 14, 455-461. van der Kolk, B. A., \& Fisler, R. (1995). Dissociation and the fragmentary nature of traumatic memories: Overview and exploratory study. Journal of Traumatic Stress, 8, 505-525.

Wittmann, L., Moergeli, H., \& Schnyder, U. (2006). Low predictive power of peritraumatic dissociation for PTSD symptoms in accident survivors. Journal of Traumatic Stress, 19, 639-651.

Yadin, E., \& Foa, E. B. (2007). Cognitive behavioral treatments for posttraumatic stress disorder. In L. J. Kirmayer, R. Lemelson, \& M. Barad (Eds.), Understanding trauma: Integrating biological, clinical, and cultural perspectives (pp. 178-193). New York, NY: Cambridge University Press.

Yates, J. L., \& Nasby, W. (1993). Dissociation, affect, and network models of memory: An integrative proposal. Journal of Traumatic Stress, 6, 305326

Zoellner, L. A., Jaycox, L. H., Watlington, C. G., \& Foa, E. B. (2003). Are the dissociative criteria in ASD useful? Journal of Traumatic Stress, 16, 341-350. 